\title{
Maternal inheritance of mitochondria: implications for male fertility?
}

\author{
R C Vaught and D K Dowling \\ School of Biological Sciences, Monash University, Clayton, Victoria, Australia \\ Correspondence should be addressed to R C Vaught or D K Dowling; Email: rebecca.vaught@monash.edu \\ ordamian.dowling@monash.edu
}

\begin{abstract}
Evolutionary theory predicts maternal inheritance of the mitochondria will lead to the accumulation of mutations in the mitochondrial DNA (mtDNA) that impair male fertility, but leave females unaffected. The hypothesis has been referred to as 'Mother's

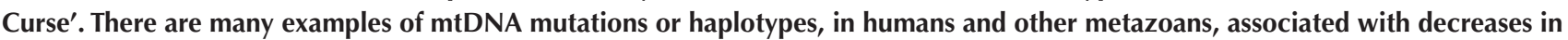
sperm performance, but seemingly few reports of associations involving female reproductive traits; an observation that has been used to support the Mother's Curse hypothesis. However, it is unclear whether apparent signatures of male bias in mitochondrial genetic effects on fertility reflect an underlying biological bias or a technical bias resulting from a lack of studies to have screened for female effects. Here, we conduct a systematic literature search of studies reporting mitochondrial genetic effects on fertility-related traits in gonochoristic metazoans (animals with two distinct sexes). Studies of female reproductive outcomes were sparse, reflecting a large technical sex bias across the literature. We were only able to make a valid assessment of sex specificity of mitochondrial genetic effects in $\mathbf{3 0} \%$ of cases. However, in most of these cases, the effects were male biased, including examples of male bias associated with mtDNA mutations in humans. These results are therefore consistent with the hypothesis that maternal inheritance has enriched mtDNA sequences with mutations that specifically impair male fertility. However, future research that redresses the technical imbalance in studies conducted per sex will be key to enabling researchers to fully assess the wider implications of the Mother's Curse hypothesis to male reproductive biology.

Reproduction (2018) 155 R159-R168
\end{abstract}

\section{Introduction}

Mitochondria are thought to have evolved from an ancient union between an $\alpha$-proteobacterium and archaean eukaryote ancestor (Gray et al. 1999); an endosymbiotic event that enabled the ancestral singlecell eukaryote to harness an extremely efficient means of energy conversion via oxidative phosphorylation (OXPHOS). Indeed, the mitochondria are intriguing from an evolutionary standpoint, because they have retained their own genome, which has been vastly streamlined throughout the course of eukaryote evolution via translocation of hundreds of genes required for mitochondrial functionality across to the nuclear genome. What remains in the mitochondrial DNA (mtDNA) of metazoans is a 15-20 kilobase sequence of 37 genes, of which just 13 encode proteins. The evolutionary implication is that the production of ATP, the key energy currency on which the integrity of eukaryotic life relies, depends on a coordinated set of interactions between genes that reside across two obligate genomes (Fig. 1) - mitochondrial and nuclear (Rand et al. 2004, Wolff et al. 2014).

\section{The mitochondrial genotype-phenotype linkage}

It was long-assumed by evolutionary and population geneticists that any genetic polymorphisms segregating within the mtDNA sequence would have negligible, if any, effect on organismal phenotypes, and result in no modifications to traits such as development rate, fertility or life expectancy (Ballard \& Kreitman 1995, Galtier 2009). This notion was based on the assumption that the products of the mitochondrial genome (polypeptide subunits directly involved in OXPHOS function) are so integral to sustaining the viability of eukaryotic life, that natural selection would act swiftly to purge any de novo mutations appearing in the mtDNA sequence; leaving only a pool of residual genetic variation in the genome that was 'neutral' to selection, and of no consequences to health (Avise et al. 1987, Dowling et al. 2008).

In recent years, however, numerous studies have emerged that used experimental approaches to experimentally uncouple mitochondrial from nuclear genetic contributions to the expression of organismal phenotypes, in a range of invertebrate and vertebrate metazoans. These studies have routinely linked the 


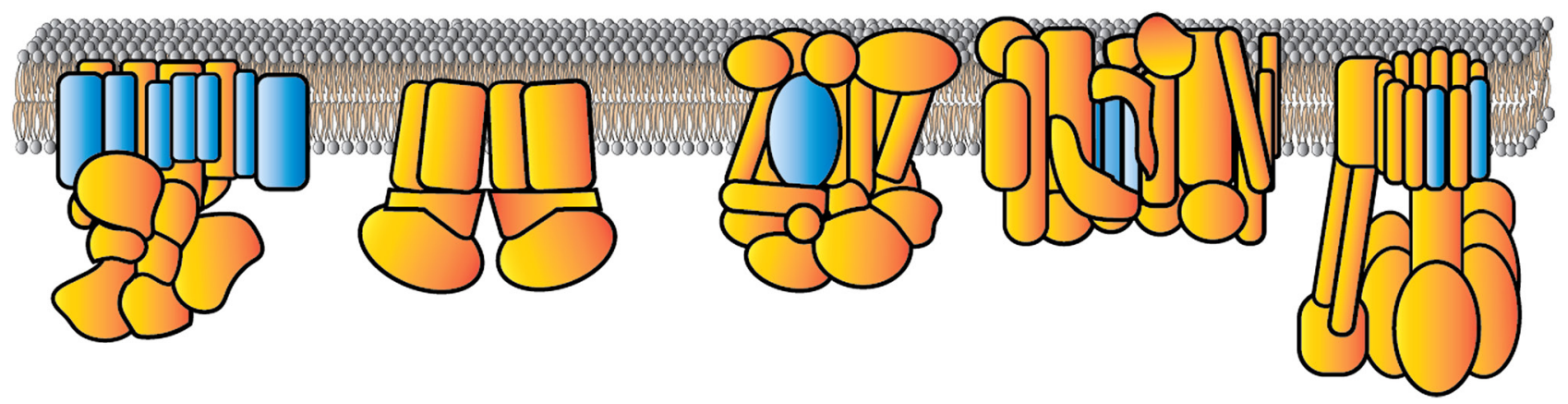

Figure 1 Schematic depiction of the enzyme complexes of the oxidative phosphorylation (OXPHOS) chain located within the mitochondrial inner membrane. Subunits in yellow are encoded by the nuclear genome, subunits in blue encoded by the mitochondrial genome. ATP production thus relies on the function of five enzymes, four of which depend on coordinated interactions involving both nuclear- and mitochondrial-encoded subunits.

genetic variation that delineates naturally occurring 'mtDNA haplotypes' to modifications in a range of phenotypes related to health, such as metabolism, growth and longevity, in both invertebrate (Rand 2001, Meikeljohn et al. 2007, Camus et al. 2012, 2015, Yee et al. 2013) and vertebrate metazoans (Fontanillas et al. 2005, Smith et al. 2010, Boratyński et al. 2016). Many of these papers have been reviewed elsewhere (Ballard \& Kreitman 1995, Blier 2001, Ballard \& Whitlock 2004, Ballard \& Rand 2005, Dowling et al. 2008, Ballard \& Pichaud 2014, Dobler et al. 2014, Dowling 2014) and will not be discussed here. Coupled with these findings, it has become increasingly clear that diseasecausing mtDNA mutations might commonly exist at appreciable frequencies within human populations (Chinnery et al. 2000, Taylor \& Turnbull 2005, Reeve et al. 2008), with around one in 200 people estimated to carry a pathogenic mtDNA mutation (Elliott et al. 2008), and around one in 5000 ultimately expressing a mitochondrial disease phenotype at some stage of their lives (Thorburn 2004). Furthermore, different mtDNA variants have recently been implicated in the penetrance or severity of a range of late-onset human diseases that were previously thought to be unrelated to mitochondrial function, from schizophrenia, to multiple sclerosis and Parkinson's disease (Hudson et al. 2014). Thus, it is becoming apparent that the genetic variation that accumulates within the mtDNA sequences of natural populations of metazoans does play an important role in determining health outcomes, by contributing to metabolic health, development trajectories, lifespan and the risk of disease progression (Wallace 2005, Dowling et al. 2008, Reinhardt et al. 2013, Dowling 2014).

\section{Theory linking the mitochondria to male fertility}

In 1996, Frank and Hurst formulated an evolutionary hypothesis based on a simple population genetic model that predicts that mitochondrial genomes will be enriched for mutations that impair male fertility. Gemmell et al. (2004) called the hypothesis 'Mother's Curse'; a name we adopt here given it has since been used by the majority of studies that address this hypothesis (Wade \& Brandvain 2009, Smith et al. 2010, Hedrick 2011, Zhang et al. 2012, Wade 2014). The mitochondria are strictly maternally inherited in metazoans, with males rarely, if ever, transmitting their mtDNA to their offspring. Thus, males are effectively evolutionary dead-ends when it comes to mtDNA transmission, and as a result 'natural selection' should only be effective at shaping evolutionary changes within the mitochondrial genome through females (i.e. when the genome is carried inside of females). That is, if a mutation arises in the mtDNA sequence that is relatively neutral in its effects on females, this mutation will evade selection and could accumulate within populations, even if the effects of the very same mutation are negative for males (Frank \& Hurst 1996, Innocenti et al. 2011, Frank 2012, Beekman et al. 2014). In theory, mtDNA mutations might even arise that augment female function, but decrease male function. In such cases, these 'male-harming' mtDNA mutations would be expected to increase in their frequency within a population under positive Darwinian selection, given that evolutionary adaptation of the mtDNA sequence proceeds through females (Beekman et al. 2014).

While the Mother's Curse hypothesis is theoretically sound, its biological relevance necessarily rests on an assumption that mutations that confer sex-specific effects can and do accumulate within the mtDNA sequence. Below, we address the capacity for such sex specificity. The genetic correlation between the sexes is expected to be strongly positive for most traits (Bonduriansky \& Chenoweth 2009). This is because the sexes share the same genomes, both nuclear and mitochondrial (Pennell \& Morrow 2013), and the genes encoding trait function are likely to have much the same role in each of the sexes. Thus, if a mutation was to arise in a mitochondrial gene that encodes a critical element of OXPHOS function and has a negative effect on males, then it is reasonable to assume that under most scenarios, this same mutation would exert a similarly negative effect on females. Selection would then operate on females 
to remove this mtDNA mutation from the population (i.e., females that carry it would be less likely to pass on their mtDNA), and males of future generations would salvage the benefits of this female-mediated optimisation of the mtDNA sequence. Accordingly, when it comes to the optimisation of mitochondrial function, males should generally be able to depend on natural selection operating through females, to meet their metabolic requirements.

Biological research, however, has provided many examples of sexual dimorphism in trait expression (Karp et al. 2017). Indeed, there are no traits that exhibit greater differences between the sexes than those tied directly to reproduction - the gonads and gametes. The male homologs of these traits (the testes and sperm) exhibit high metabolic activity (Ewing et al. 1966, Short 1997), while their female counterparts (ovaries and ova) are relatively quiescent (Short 1997). Indeed, in vertebrates, the capacity of sperm to fertilise the ova depends primarily on their motility, powered by a small number of mitochondria packed within the sperm midpiece. It seems plausible to assume that the metabolic requirements of the testes and sperm must to some degree depend on products of the mtDNA. Yet, paradoxically, when it comes to mtDNA-mediated optimisation of gonad and gamete function (via natural selection), this will in theory proceed through selection of mtDNA sequence variants that maximise performance in the ovaries and ova; and this could lead to the accumulation of mtDNA variants that while optimised for function in the female gonads and gametes, confer suboptimal function in the male homologs.

Furthermore, even in the absence of mtDNA mutations that directly encode sex-specific reproductive effects, mtDNA-mediated sex specificity in gamete function could plausibly arise through a second mechanism. The capacity for sex-general mtDNA mutations (mutations that exert similar pathogenicity in both females and males) to accumulate to appreciably high frequencies in the gametes is greater in males than in females, due to vast discrepancies in the mitochondrial content of the sperm and ova. The mature oocyte may contain hundreds of thousands of mitochondria in humans (Shoubridge \& Wai 2007), whereas the mature sperm carries less than 100 (Ankel-Simmons \& Cummins 1996). Mutations in the mtDNA will generally only confer pathogenicity when their frequency within a cell lineage surpasses a biochemical threshold of mutant-towild type molecules. This threshold is typically around $70-80 \%$, although varies across tissue types (Wallace 1999). Such thresholds for a mutant mtDNA molecule within the ovum might be extremely difficult to surpass by stochastic processes alone (the random segregation of mutant-to-wild-type mtDNA molecules) given the high copy number of mtDNA in this cell, but might be more routinely reached in the sperm in which there are very few copies of mtDNA (Gemmell et al. 2004).
Accordingly, the male gamete is predicted to be much more sensitive to the effects of mtDNA mutations than its female counterpart. In fact, in theory, the accumulation of male fertility-impairing mutations in the mtDNA sequence should in itself select for a variety of evolutionary mechanisms that offset the negative effects to males. Such mechanisms might include genetic adaptations that occasionally permit paternal leakage in transmission of mtDNA at conception, thereby enabling male-harming haplotypes associated with particular ancestral matrilines to be replaced (Kuijper et al. 2015, Radzvilavicius et al. 2017). Alternatively, the presence of male fertility-impairing mutations in the mtDNA should invoke selection on the nuclear genome for compensatory counter-adaptations able to restore male fertility (Beekman et al. 2014, Connallon et al. 2017). However, while instances of paternal transmission have been previously observed in metazoans, these are typically only observed at low rates (Bromham et al. 2003, White et al. 2008, Wolff et al. 2013, Dokianakis \& Ladoukakis 2014) or when involving reproductive events between very distantly related genetic lineages. Similarly, when it comes to evidence for compensatory capacities of nuclear genotypes that have coevolved to offset male-harming mutations, empirical evidence for such counter-adaptations remains scarce in metazoans (Yee et al. 2013, Wolff et al. 2017).

Indeed, over the past two decades, several studies have reported links between mitochondrial genetic variation and negative male reproductive outcomes (Gemmell et al. 2004), suggesting that the evolution of compensatory mechanisms is, at best, only partly effective in offsetting the effects of male-harming mtDNA mutations. Moreover, there appears to be a sex bias emerging in the reporting of such effects, with numerous studies suggesting that different mtDNA variants affect components of male fertility (Folgerø et al. 1993, Kao et al. 1998, Ruiz-Pesini et al. 2000a,b, Froman \& Kirby 2005, Montiel-Sosa et al. 2006, Colagar et al. 2013, Yee et al. 2013, Tourmente et al. 2017), but seemingly less reporting similar effects in females (Nakada et al. 2006, Xu et al. 2008, Dowling et al. 2009, Smith et al. 2010, Patel et al. 2016). These studies have therefore generated attention because they appear consistent with the predictions of the Mother's Curse hypothesis. However, it is critical to note that although such sex biases might reflect true biological differences between the sexes in the fertility effects conferred by different mtDNA variants, they could alternatively reflect a broader sex bias in the design and implementation of animal experiments. Indeed, there has been a historical trend for researchers in the animal sciences to focus on male subjects in their scientific research (Zucker \& Beery 2010, Karp et al. 2017). One of the implications of this bias in the use of male subjects is that it could then create an illusion of biological sex bias and lead to misinterpretations of biological processes, given the 
sex biases might be based on an absence of female data, rather than an absence of effects per se in females.

Therefore, the primary goal of this study was to provide an up-to-date, systematic review of the effects of mtDNA variation on components of reproduction in gonochoristic metazoans (animals with two distinct sexes), to enable us to appraise two questions; firstly, whether previously reported male biases in mitochondrial genetic effects on fertility are pervasive across metazoans (from invertebrates to humans), and secondly whether any such biases reflect true sex biases in nature or a sampling (technical) bias by animal researchers. Evidence for the former requires that fertility effects of particular mtDNA variants or haplotypes in males are corroborated by parallel evidence of null effects in females.

\section{Evidence for sex-specific mitochondrial fertility effects}

We conducted a comprehensive literature search of papers reporting mitochondrial and cytoplasmic effects on components of reproduction in animals, using the Web of Science, and by screening reference lists of key review articles in the subject area. The final Web of Science search was adapted from Dobler et al. (2014) and contained the terms for the field 'topic' as follows:

((cytop* OR mitoch* OR cytoty* OR mitoty* OR mtDNA* OR cybrid* OR 'mtDNA mutation*' OR 'mtDNA varia*' OR 'mtDNA deletion*' OR 'mitochondrial deletion*' OR 'mitochondrial mutation*' OR 'mitochondrial genom*' OR 'mtDNA polymorphi*' OR 'mtDNA snp*' OR 'mitochondrial polymorphi*' OR 'mitochondrial snp*' OR 'mitochondrial DNA*' OR 'mitochondrial DNA hypomorph*' OR 'mtDNA hypomorph*' OR haplotype* OR haplogroup* OR 'Mother's Curse' OR 'Frank and Hurst') AND (varia* OR effect* OR affect* OR interaction) NOT cance* NOT neuro* NOT phylogeo* NOT bacter*) AND (reprod* OR fertility* OR fecund* OR sperm* OR infertil* OR fertil* OR oligozoosperm* OR asthenozoosperm* OR 'reproductive senescence' OR 'reproductive ag*' OR ejacul* OR testes* OR semen* OR semin* OR 'mating* rate').

This search was further limited by including only primary literature and by excluding irrelevant fields. The fields included in the search were limited to the following: genetics, heredity, evolutionary biology, reproductive biology, ecology, biochemistry, molecular biology, cell biology, zoology, biology, developmental biology, multidisciplinary sciences, andrology, entomology, agriculture, dairy animal science, marine biology and freshwater biology.

The literature search returned 4175 results in total, which were individually screened for content by RCV. Of the 53 papers assessed as relevant to our study, 38 comprised studies of individual mutations or sets of mutations (category 1: mutation studies), in which researchers screened for associations between known mtDNA mutations and traits associated with fertility, such as metrics of sperm performance or reproductive outcomes per se. For each of these mtDNA mutations, or set of mutations examined, we could assess whether associated fertility effects had been tested in each of the sexes (i.e., not just in males), and if so, whether the reported effects of the mutations on the reproductive phenotypes were negative, positive or neutral. Another 15 studies utilised 'panels' of mtDNA haplotypes (category 2: panel studies) to screen for effects of natural variation across mtDNA haplotypes on fertilityrelated traits (sperm or reproductive traits). These studies come in two types. One subset was screened for associations between mtDNA haplotypes and sperm or reproductive traits, but was not controlled for segregating variation in the nuclear background. These are by definition association studies. The other subset utilised experimental approaches to place a defined set of naturally occurring mtDNA haplotypes alongside a standardised nuclear genetic background, thus allowing effects of mitochondrial genetic variation to be directly mapped to the reproductive traits involved. In studies of panels, it is generally not possible to ascribe directionality to the effects of the mtDNA haplotypes on the fertility-related traits, because each study tests the effects of many different mtDNA haplotypes on the traits rather than individual candidate mutations. Rather, here we simply record whether the studies found associations (Yes/No) between mitochondrial haplotype variation and fertility-related traits in either sex.

We focus on the identity of the mtDNA mutation or mutation set in category 1 studies, and the identity of the panel in category 2 studies, as our units of inference rather than the individual study/paper. This is because some mutations/panels have been the focus of multiple studies. This is essential to avoid issues of pseudoreplication (i.e., numerous studies of the same mutation/set of mutations/ panel) from confounding our interpretations. We have collated the data on phenotypic effects associated with these mutations/panels on traits associated with male fertility, female fertility or on other non-reproductive traits (e.g. mitochondrial disease phenotypes, respiratory capacity, longevity, etc.) (Supplementary Table 1, see section on Supplementary data given at the end of this article). In total, we collated phenotypic effects associated with 30 different mutations/sets, and 10 different panels, spanning 10 different species. Of these, only $30 \%$ of mutations/mutation sets/panels contained data for both sexes ( 7 of 30 for mutations/sets, and 5 of 10 for panels). Of the mutations/mutation sets/panels that contained data for both males and females (i.e., of the cases that were not affected by a technical sex bias), 10 of 12 exhibited signatures of male bias in the presence and/or severity of the reported effects (6/7 for mutations/sets and $4 / 5$ for panels). 
From these results, it is clear that a large technical sex bias exists within the literature. Most mutations/ mutation sets/panels contain data for only one sex, usually males. Furthermore, of the 28 mutations/ mutation sets/panels exhibiting a technical sex bias, only 5 of these had been created and studied with the explicit goal of testing the Mother's Curse hypothesis. Thus, the technical bias persists because of other factors, such as the ease by which male reproductive traits, such as sperm parameters, can be measured relative to their female counterparts (ova quality), the latter of which might be more routinely monitored through non-invasive and indirect screens of hormone levels and ovulation. Moreover, we note that it is possible that other studies that tested effects of mtDNA mutations on components of reproduction in males or females might have withheld results from publication, if their results were in line with the null hypothesis (a phenomenon known as the 'file drawer' problem). Such withholding of null results from the literature could in theory influence interpretations of the dataset that we have collated. Our study is the first to systematically quantify a glaring technical sex bias when it comes to mitochondrial genetic effects on components of reproduction.

Notwithstanding this large technical bias, we observed a clear signature of biological male bias in effects for 10 of the 12 mutations/mutations sets/ panels in which data existed for both males and female components of reproduction. This result is consistent with the evolutionary hypothesis of Mother's Curse and reinforces the importance of future studies redressing the technical bias identified above, to enable a full appreciation of the generality by which mutations in the mtDNA might confer sex differences in reproductive outcomes across metazoans. Currently, most of the data for Mother's Curse effects on reproduction comes from humans and two well-studied model organisms (Drosophila and Mus), and we specifically discuss these case studies below.

\section{Fruit fly case studies}

All of the fruit fly examples come from studies of $D$. melanogaster, and many of these have utilised the same panel of naturally occurring mtDNA haplotypes (Clancy et al. 2008, 2011, Innocenti et al. 2011, Yee et al. 2013, Dowling et al. 2015, Wolff et al. 2016a,b, 2017), which were originally sourced from different global localities and which exhibit generally low levels of sequence divergence $(\sim 0.4 \%)$; a level of divergence paralleling that seen between major human mtDNA haplogroups (Morrow et al. 2015). Replicate genetic strains have been created for each of these haplotypes, with each haplotype placed alongside a standardised isogenic background (i.e. a nuclear genetic background devoid of segregating allelic variation), which allowed direct estimation of the magnitude of the mitochondrial haplotype effect on a range of phenotypes. One haplotype in this panel, originally sourced from a wild population in Brownsville, Texas, USA (Rand et al. 1994), is associated with reduced male fertility, and its proteincoding sequence differs from the other haplotypes in the panel by a unique SNP within the mitochondrial cytochrome b gene of respiratory complex III, which results in an amino acid transition ( $\left.m t: C y t B^{A 278 T}\right)$. Thus, this is the candidate SNP causing the sterility, although it is possible that other currently cryptic variation in the D-loop is driving the effects (Wolff et al. 2017). These subfertility effects associated with this haplotype have been confirmed across all nuclear genetic and environmental contexts in which males harbouring this haplotype have been tested (Clancy et al. 2008, Yee et al. 2013, Dowling et al. 2015, Wolff et al. 2016a,b, 2017). Indeed, in one nuclear background in which the haplotype has been tested, the spermatids of these males fail to individualise and degrade within the testes (Clancy et al. 2011), leaving the males completely infertile. In contrast, females that carry this Brownsville haplotype, and its candidate male-sterilising $m t: C y t B$ SNP, enjoy high reproductive success typical of females carrying other wild-type haplotypes (Camus \& Dowling 2017). Thus, this Brownsville haplotype specifically depresses male, but not female, fertility. Intriguingly, the most recent study on this panel of haplotypes documented a pattern of sexual antagonism in reproductive outcomes; those haplotypes associated with the lowest reproductive success in males were the ones that conferred highest reproductive success in females. This pattern supports the notion that mitochondrial genomes accumulate mutations that confer beneficial effects in females, but negative effects on males, with these mutations accumulating under positive selection as a result of mitochondrial maternal inheritance (Unckless \& Herren 2009, Innocenti et al. 2011, Beekman et al. 2014).

Patel et al. (2016) reported another mtDNA mutation rendering male sub-fertility in $D$. melanogaster, which was found to be naturally segregating in the laboratory population of a common fruit fly strain known as $w^{1118}$. The mutation is a non-synonymous SNP, resulting in a glycine to serine substitution at position 177 in subunit II of the cytochrome c oxidase ( $m t: C O / I^{G 1775}$ ) gene of respiratory complex IV. The glycine variant at position 177 is conserved across eukaryotes. The serine variant conferred a reduction in male, but not female fertility, with the magnitude of the reduced fertility in males increasing with advancing age and also greatly exacerbated at higher temperatures.

While the former examples involve genetic polymorphisms that evolve and segregate naturally in wild or laboratory populations, further evidence for male infertility-impairing mtDNA mutations in D. melanogaster comes from an earlier study which genetically-engineered mutant mtDNA variants. Xu and coworkers (Xu et al. 2008) used targeted restriction 
enzymes to select for germ-line mtDNA mutations in subunit I of the mitochondrial cytochrome c oxidase gene. Using this approach, they identified three sequence variants, one of which possessed a normal phenotype ( $m t: \mathrm{Col}^{A 302 T}$, normal for fertility, cytochrome c oxidase activity and ATP production), while the other two conferred male sterility. More specifically, males carrying $m t: \mathrm{Col}^{R 301 Q}$ were sterile, producing low numbers of motile sperm, but were otherwise healthy, exhibiting normal cytochrome c oxidase activity and ATP levels. A fertility effect on females was not reported, but presumed absent. Males carrying $m t: \mathrm{Col}^{R 301 S}$ were also completely sterile, lacking mature sperm; while females carrying this variant also experienced a large reduction in fertility relative to their wild-type counterparts (producing 20\% the offspring of wild-types). $m t: C o l^{R 3015}$ flies also experienced greatly reduced cytochrome c oxidase activity and ATP levels (Xu et al. 2008).

In sum, in $D$. melanogaster, mutations that compromise male, but not female, fertility appear to be a feature of the mutational landscape of mitochondrial genomes, with at least three independent mutations, spanning two genes, now identified (Xu et al. 2008, Clancy et al. 2011, Dowling et al. 2015, Patel et al. 2016), and accumulating evidence of numerous other mutations of smaller effect that depress male but increase female fertility (Camus \& Dowling 2017). Evidence therefore exists in D. melanogaster that male biases in these reported cases reflect true sex biases in the manifestation of these mtDNA-mediated fertility effects and are not technical artefacts of a sex bias in design and implementation of experiments.

\section{Mouse case studies}

Studies of mice have also provided evidence that mtDNA mutations exist that confer negative effects on male, but not female, fertility. Nakada et al. (2006) studied mice strains that were heteroplasmic for a large deletion from position 7759 from the $m t: t R N A^{L y s}$ gene to position 12,454 of the $m t: N D 5$ gene. The authors reported various pathologies, the expression of which depended on the proportion of the mutant mtDNA molecules (level of heteroplasmy) per individual mouse. Males carrying less than $68 \%$ of the mutant mtDNA were healthy and fertile. Males carrying between 70 and $80 \%$ of the mutant mtDNA were also mostly healthy, exhibiting only slight respiratory deficiencies. However, although these mice produced copulation plugs, they produced fewer, less motile sperm, and consequently experienced a drastic reduction in fertility compared to control mice (as gauged by an approximate $60 \%$ decrease in the number of eggs, submitted to IVF with the experimental males' sperm, reaching the two-cell embryonic stage). Males carrying more than $80 \%$ of the mutant mtDNA type were completely infertile, failed to produce copulation plugs and produced only very few immotile sperm. These males with the highest mutant loads also exhibited widespread respiratory deficiencies, and other pathologies associated with mitochondrial disease (myopathy, lactic acidosis, renal failure, deafness). Although male fertility was particularly sensitive to the presence of this mtDNA deletion, the authors noted that females carrying more than $70 \%$ of the mutant mtDNA type still produced normal numbers of progeny, at least up until six months of age when they died of mitochondrial pathologies associated with the mutation (Inoue et al. 2000, Nakada et al. 2004).

The approach of Nakada and colleagues is complemented by two other studies in mice. Firstly, Trifunovic and colleagues (Trifunovic et al. 2004) generated mouse strains carrying mtDNA polymerase with a mutation in the catalytic PolgA subunit, rendering it proofreading deficient, and subsequently resulting in mice exhibiting a threefold to fivefold increase in the level of point mutations accumulating in their mtDNA sequences. These 'mtDNA-mutator' mice expressed normal phenotypes until around 25 weeks of age. While mtDNA-mutator mice of both sexes exhibited fertility reductions relative to wild-type mice, the magnitude of reduction was again male biased. Almost all mtDNAmutator females produced one or two litters of normal size up until the age of 20 weeks, at which point they were no longer reproductive. Mutator males, however, were mostly infertile, with only one of eight males tested producing a litter; and mtDNA-mutator males possessed testes of much smaller size relative to wild-type males, even early in adult life (at 12 weeks). Secondly, Ma et al. (2016) created reciprocal (conplastic) combinations of mitochondrial and nuclear genotypes in the zygotes of two divergent mice strains; Mus $m$. domesticus (B6 strain) and Mus m. musculus (PWD strain) mice. Male offspring carrying PWD mtDNA in a B6 nuclear autosomal background exhibited reduced fertility, as gauged by number of litters produced, but females were unaffected. This male infertility effect is likely attributable to mutations in the mtDNA sequence of the PWD mice, which are normally rescued by modifier mutations that lie within the PWD nuclear background, but which were not present in the experimental mice carrying the B6 background (Ma et al. 2016).

In sum, there have been three studies in mice that have examined effects of mtDNA variation on reproductive outcomes of both sexes (Inoue et al. 2000, Nakada et al. 2006, Ma et al. 2016). Each of these studies has documented male biases in fertility reductions that map to mutations in the mitochondrial genome. Again, consistent with the Drosophila case studies reviewed above, the studies on mice provide empirical support for the tenet that mtDNA sequences are enriched for mutations that specifically depress male components of reproduction. 


\section{Human case studies}

Unlike the Drosophila and mouse studies, which are able to leverage experimental approaches to partition mitochondrial genetic, from nuclear genetic and environmental effects on fertility outcomes, human studies must rely principally on inferences drawn from correlations between mtDNA variants and fertility outcomes. Various associations have been reported between naturally occurring mtDNA haplogroups and incidences of male infertility. Ruiz-Pesini et al. $(2000 a, b)$ reported that the human $T$ haplogroup is overrepresented among males with low sperm motility, while the $\mathrm{H}$ haplogroup is overrepresented among males with high motility. Montiel-Sosa et al. (2006) reported associations between distinct sub-haplogroups within the $U$ haplogroup and variation in sperm motility and attempted to map these patterns to particular polymorphisms that distinguish the sub-haplogroups. Various other studies linked particular point mutations or deletions in human mtDNA to sperm dysfunction (Folgerø et al. 1993, Huang et al. 1994, Kao et al. 1995, Fadic et al. 1997, Lestienne et al. 1997, Kao et al. 1998, Holyoake et al. 2001, St John et al. 2001, Spiropoulos et al. 2002, Thangaraj et al. 2003, Kumar et al. 2009, Baklouti-Gargouri et al. 2013a,b, Colagar et al. 2013, Chari et al. 2015, Lu et al. 2015). Yet, some caution must be applied to interpreting correlations of the type presented in these studies, since the patterns could be mediated by other confounding factors, such as differences in nuclear genetic structure between groups of individuals possessing the different mtDNA haplotypes. Furthermore, it remains unknown whether similar correlations exist between these human haplotypes and candidate mutations to patterns of female fertility.

However, in a recent development, Martikainen and colleagues (Martikainen et al. 2017) analysed the reproductive success of males drawn from a database of British patients suffering mitochondrial disease and reported that reproductive success of these males was only $65 \%$ of that of males from the general population. The magnitude of the effect on reproductive success increased with the severity of the mitochondrial disease symptoms in these males. The effects on fertility were equally evident among the cohort of males whose disease symptoms were caused by pathogenic mutations in the mtDNA or mutations in nuclear genes that encode mitochondrial function. Many of the patients suffering from mtDNA-mediated mitochondrial disease in this study carried the m.3243A>G mutation, which is associated with a large range of mitochondrial disease symptoms (Brown \& Wallace 1994) and has been previously associated with low sperm motility (Spiropoulos et al. 2002). Remarkably, these patterns linking mtDNA mutations to reduced reproductive success were not found in female patients of the same database carrying pathogenic mtDNA mutations
(Gorman et al. 2015). Generally, little is known about putative reproductive effects in females carrying the m.3243A > G mutation, other than an investigation of a single Chinese family carrying this mutation, some of the females of which experienced frequent miscarriages (Huang et al. 1994).

In sum, the recent evidence in humans is consistent with the evolutionary hypothesis prediction that mitochondrial genomes will harbour mutations that confer male biases in reproductive outcomes and aligns with the experimental evidence acquired from the study of Drosophila and mice. Future association studies in humans should, however, focus on the possible effects of the mutations under study on components of female reproductive success, to redress what appears to be a sex bias in the study of human mtDNA variation and its effects on fertility.

\section{Conclusion}

Evolutionary theory predicts that maternal inheritance of the mitochondria will lead to the accumulation of mtDNA mutations that confer male-specific effects on fertility (Frank \& Hurst 1996). Yet, until now, a systematic investigation of Mother's Curse effects across metazoans has not been conducted, and evidence for Mother's Curse has largely remained theoretical (Frank \& Hurst 1996, Gemmell et al. 2004) or limited to a few prominent empirical examples (Smith et al. 2010, Patel et al. 2016). Our literature search revealed a striking technical sex bias in research examining effects of mtDNA mutations on reproductive outcomes. However, even when excluding case studies affected by technical bias from further consideration, the majority of the remaining cases (over $80 \%$ ) exhibited male biases in the magnitude of mitochondrial genetic effects on fertility, suggestive of Mother's Curse effects. We have focused our discussion on robust case studies from Drosophila, mice and humans, because these encompass the vast majority of studies conducted to date and are underscored by reproductive data collected from both sexes. We note, however, that these case studies are supported in several cases by intriguing patterns of male bias in mitochondrial effects on fertility in nonmodel species (Smith et al. 2010). Taken together, these findings suggest that mitochondrial genomes might be enriched for male fertility-impairing mutations across metazoans.

Yet, challenges lie ahead before such a suggestion can be confirmed, and the most obvious is the need to redress the large technical sex bias in research linking the mtDNA to fertility outcomes. For example, almost all of the association studies conducted in humans, which have studied the effects of particular mtDNA haplotypes or mutations on reproductive performance, focused exclusively on males. As such, while several cases of 
male-specific mtDNA-mediated infertility have now been confirmed across model species, it remains unclear whether these cases represent anomalies specific to a few mtDNA mutations appearing in one or a few animal species or whether they represent a small fraction of the total male-biased mtDNA variants that might be segregating in animal mitochondrial genomes. We believe the coming years will provide crucial resolution of these questions, inspired by emerging research that suggests a role for the Mother's Curse hypothesis in human reproductive biology (Dowling 2014, Milot et al. 2017).

\section{Supplementary data}

This is linked to the online version of the paper at https://doi.org/10.1530/REP-17-0600.

\section{Declaration of interest}

The authors declare that there is no conflict of interest that could be perceived as prejudicing the impartiality of this review.

\section{Funding}

This study was funded by the Australian Research Council (FT160100022 and DP170100165) to D K D.

\section{Acknowledgements}

The authors thank two anonymous reviewers for comments that significantly improved the paper.

\section{References}

Ankel-Simons F \& Cummins JM 1996 Misconceptions about mitochondria and mammalian fertilization: implications for theories on human evolution. PNAS 93 13859-13863. (https://doi.org/10.1073/ pnas.93.24.13859)

Avise JC, Arnold J, Ball RM, Bermingham E, Lamb T, Neigel JE, Reeb CA \& Saunders NC 1987 Intraspecific phylogeography: the mitochondrial DNA bridge between population genetics and systematics. Annual Review of Ecology and Systematics 18 489-522. (https://doi.org/10.1146/annurev. es.18.110187.002421)

Baklouti-Gargouri S, Ghorbel M, Mahmoud AB, Mkaouar-Rebai E, Cherif M, Chakroun N, Sellami A, Fakhfakh F \& Ammar-Keskes L 2013a A novel m. 6307A>G mutation in the mitochondrial COXI gene in asthenozoospermic infertile men. Molecular Reproduction and Development 80 581-587. (https://doi.org/10.1002/mrd.22197)

Baklouti-Gargouri S, Ghorbel M, Mahmoud AB, Mkaouar-Rebai E, Cherif M, Chakroun N, Chakroun N, Sellami A, Fakhfakh F \& AmmarKeskes L 2013b Mitochondrial DNA mutations and polymorphisms in asthenospermic infertile men. Molecular Biology Reports 40 4705-4712. (https://doi.org/10.1007/s11033-013-2566-7)

Ballard JWO \& Kreitman M 1995 Is mitochondrial DNA a strictly neutral marker? Trends in Ecology and Evolution 10 485-488.

Ballard JWO \& Pichaud N 2014 Mitochondrial DNA: more than an evolutionary bystander. Functional Ecology 28 218-231. (https://doi. org/10.1111/1365-2435.12177)
Ballard JWO \& Rand DM 2005 The population biology of mitochondrial DNA and its phylogenetic implications. Annual Review of Ecology, Evolution, and Systematics 36 621-642. (https://doi.org/10.1146/ annurev.ecolsys.36.091704.175513)

Ballard JW \& Whitlock MC 2004 The incomplete natural history of mitochondria. Molecular Ecology 13 729-744. (https://doi.org/10.1046/ j.1365-294X.2003.02063.x)

Beekman M, Dowling DK \& Aanen DK 2014 The costs of being male: are there sex-specific effects of uniparental mitochondrial inheritance? Philosophical Transactions of the Royal Society B 369 20130440. (https:// doi.org/10.1098/rstb.2013.0440)

Blier PU, Dufresne F \& Burton RS 2001 Natural selection and the evolution of mtDNA-encoded peptides: evidence for intergenomic co-adaptation. Trends in Genetics 17 400-406. (https://doi.org/10.1016/S01689525(01)02338-1)

Bonduriansky R \& Chenoweth SF 2009 Intralocus sexual conflict. Trends in Ecology and Evolution 24 280-288. (https://doi.org/10.1016/j. tree.2008.12.005)

Boratyński Z, Ketola T, Koskela E \& Mappes T 2016 The sex specific genetic variation of energetics in bank voles, consequences of introgression? Evolutionary Biology 43 37-47. (https://doi.org/doi:10.1007/s11692015-9347-2)

Bromham L, Eyre-Walker A, Smith NH \& Smith JM 2003 Mitochondrial Steve: paternal inheritance of mitochondria in humans. Trends in Ecology and Evolution 18 2-4.

Brown MD \& Wallace DC 1994 Molecular basis of mitochondrial DNA disease. Journal of Bioenergetics and Biomembranes 26 273-289. (https://doi.org/10.1007/BF00763099)

Camus MF \& Dowling DK 2017 Mitochondrial Genetic Effects on Reproductive Success: Signatures of Positive Intra-Sexual, but Negative Inter-Sexual Pleiotropy. bioRxiv. (https://doi.org/10.1101/138180)

Camus MF, Clancy DJ \& Dowling DK 2012 Mitochondria, maternal inheritance, and male aging. Current Biology 22 1717-1721. (https:// doi.org/10.1016/j.cub.2012.07.018)

Camus MF, Wolf JB, Morrow EH \& Dowling DK 2015 Single nucleotides in the mtdna sequence modify mitochondrial molecular function and are associated with sex-specific effects on fertility and aging. Current Biology 25 2717-2722. (https://doi.org/10.1016/j.cub.2015.09.012)

Chari MG, Colagar AH \& Bidmeshkipour A 2015 A novel large-scale deletion of the mitochondrial DNA of spermatozoa of men in north Iran. International Journal of Fertility and Sterility 8 453. (https://doi. org/10.22074/ijfs.2015.4185)

Chen T, He J, Shen L, Fang H, Nie H, Jin T, Wei X, Xin Y, Jiang Y, Li H et al. 2011 The mitochondrial DNA 4977-bp deletion and its implication in copy number alteration in colorectal cancer. BMC Medical Genetics 12 8. (https://doi.org/10.1186/1471-2350-12-8)

Chinnery PF, Johnson MA, Wardell TM, Singh-Kler R, Hayes C, Brown DT, Taylor RW, Bindoff LA \& Turnbull DM 2000 The epidemiology of pathogenic mitochondrial DNA mutations. Annals of Neurology 48 188-193. (https://doi.org/10.1002/1531-8249(200008)48:2<188::AIDANA8>3.0.CO;2-P)

Clancy DJ 2008 Variation in mitochondrial genotype has substantial lifespan effects which may be modulated by nuclear background. Aging Cell 7 795-804. (https://doi.org/10.1111/j.1474-9726.2008.00428.x)

Clancy DJ, Hime GR \& Shirras AD 2011 Cytoplasmic male sterility in Drosophila melanogaster associated with a mitochondrial CYTB variant. Heredity 107 374. (https://doi.org/10.1038/hdy.2011.12)

Colagar AH, Karimi F \& Jorsaraei SGA 2013 Correlation of sperm parameters with semen lipid peroxidation and total antioxidants levels in astheno-and oligoasheno-teratospermic men. Iranian Red Crescent Medical Journal 15 780. (https://doi.org/10.5812/ircmj.6409)

Connallon T, Camus MF, Morrow EH \& Dowling DK 2017 Coadaptation of mitochondrial and nuclear genes, and the cost of mother's curse. Proceedings of the Royal Society B: Biological Sciences 28520172257. (https://doi.org/10.1098/rspb.2017.2257)

Dobler R, Rogell B, Budar F \& Dowling DK 2014 A meta-analysis of the strength and nature of cytoplasmic genetic effects. Journal of Evolutionary Biology 27 2021-2034. (https://doi.org/10.1111/jeb.12468)

Dokianakis E \& Ladoukakis ED 2014 Different degree of paternal mtDNA leakage between male and female progeny in interspecific Drosophila crosses. Ecology and Evolution 4 2633-2641. (https://doi.org/10.1002/ ece3.1069) 
Dowling DK 2014 Evolutionary perspectives on the links between mitochondrial genotype and disease phenotype. Biochimica et Biophysica Acta (BBA): General Subjects 1840 1393-1403. (https://doi. org/10.1016/j.bbagen.2013.11.013)

Dowling D, Friberg U \& Lindell J 2008 Evolutionary implications of nonneutral mitochondrial genetic variation. Trends in Ecology and Evolution 23 546-554. (https://doi.org/10.1016/j.tree.2008.05.011)

Dowling DK, Maklakov AA, Friberg U \& Hailer F 2009 Applying the genetic theories of ageing to the cytoplasm: cytoplasmic genetic covariation for fitness and lifespan. Journal of Evolutionary Biology 22 818-827. (https:// doi.org/10.1111/j.1420-9101.2009.01692.x)

Dowling DK, Tompkins DM \& Gemmell NJ 2015 The Trojan Female Technique for pest control: a candidate mitochondrial mutation confers low male fertility across diverse nuclear backgrounds in Drosophila melanogaster. Evolutionary Applications 8 871-880. (https://doi. org/10.1111/eva.12297)

Elliott HR, Samuels DC, Eden JA, Relton CL \& Chinnery PF 2008 Pathogenic mitochondrial DNA mutations are common in the general population. American Journal of Human Genetics 83 254-260. (https:// doi.org/10.1016/j.ajhg.2008.07.004)

Ewing LL, Baird ER \& Vandemark NL 1966 Comparative metabolic activity of testis and kidney cortex slices of normal and hypoglycemic chickens and rabbits. Comparative Biochemistry and Physiology 17 455-465. (https://doi.org/10.1016/0010-406X(66)90580-9)

Fadic R, Russell JA, Vedanarayanan VV, Lehar M, Kuncl RW \& Johns DR 1997 Sensory ataxic neuropathy as the presenting feature of a novel mitochondrial disease. Neurology 49 239-245. (https://doi.org/10.1212/ WNL.49.1.239)

Folgerø T, Bertheussen K, Lindal S, Torbergsen T \& Øian P 1993 Andrology: mitochondrial disease and reduced sperm motility. Human Reproduction 8 1863-1868. (https://doi.org/10.1093/oxfordjournals. humrep.a137950)

Fontanillas P, Depraz A, Giorgi MS \& Perrin N 2005 Nonshivering thermogenesis capacity associated to mitochondrial DNA haplotypes and gender in the greater white-toothed shrew, Crocidura russula. Molecular Ecology 14 661-670. (https://doi.org/10.1111/j.1365-294X.2004.02414.x)

Frank SA 2012 Evolution: mitochondrial burden on male health. Current Biology 22 R797-R799. (https://doi.org/10.1016/j.cub.2012.07.066)

Frank SA \& Hurst LD 1996 Mitochondria and male disease. Nature 383 224. (https://doi.org/10.1038/383224a0)

Froman DP \& Kirby JD 2005 Sperm mobility: phenotype in roosters (Gallus domesticus) determined by mitochondrial function. Biology of Reproduction 72 562-567. (https://doi.org/10.1095/ biolreprod.104.035113)

Galtier N, Nabholz B, Glémin S \& Hurst GDD 2009 Mitochondrial DNA as a marker of molecular diversity: a reappraisal. Molecular Ecology 18 4541-4550. (https://doi.org/10.1111/j.1365-294X.2009.04380.x)

Gemmell NJ, Metcalf VJ \& Allendorf FW 2004 Mother's curse: the effect of mtDNA on individual fitness and population viability. Trends in Ecology and Evolution 19 238-244. (https://doi.org/10.1016/j.tree.2004.02.002)

Gorman GS, Grady JP, Ng Y, Schaefer AM, McNally RJ, Chinnery PF, Yu-Wai-Man P, Herbert M, Taylor RW, McFarland R et al. 2015 Mitochondrial donation - how many women could benefit? New England Journal of Medicine 372 885-887. (https://doi.org/10.1056/ NEJMc1500960)

Gray MW, Burger G \& Lang BF 1999 Mitochondrial evolution. Science 283 1476-1481. (https://doi.org/10.1126/science.283.5407.1476)

Hedrick PW 2011 Reversing Mother's Curse revisited. Evolution 66 612-616. (https://doi.org/10.1111/j.1558-5646.2011.01465.x)

Holyoake AJ, McHugh P, Wu M, O'Carroll S, Benny P, Sin IL \& Sin FYT 2001 High incidence of single nucleotide substitutions in the mitochondrial genome is associated with poor semen parameters in men. International Journal of Andrology 24 175-182. (https://doi.org/10.1046/j.13652605.2001.00292.x)

Huang CC, Chen RS, Chen CM, Wang HS, Lee CC, Pang CY, Hsu HS, Lee HC \& Wei YH 1994 MELAS syndrome with mitochondrial tRNA (Leu (UUR)) gene mutation in a Chinese family. Journal of Neurology, Neurosurgery and Psychiatry 57 586-589. (https://doi.org/10.1136/ jnnp.57.5.586)

Hudson G, Gomez-Duran A, Wilson IJ \& Chinnery PF 2014 Recent mitochondrial DNA mutations increase the risk of developing common late-onset human diseases. PLoS Genetics 10 e1004369. (https://doi. org/10.1371/journal.pgen.1004369)

Innocenti P, Morrow EH \& Dowling DK 2011 Experimental evidence supports sex-specific selective sieve in mitochondrial genome evolution. Science 332 845-848. (https://doi.org/10.1126/science.1201157)

Inoue K, Nakada K, Ogura A, Isobe K, Goto YI, Nonaka I \& Hayashi JI 2000 Generation of mice with mitochondrial dysfunction by introducing mouse mtDNA carrying a deletion into zygotes. Nature Genetics 26 176-181. (https://doi.org/10.1038/82826)

Kao S, Chao HT \& Wei YH 1995 Mitochondrial deoxyribonucleic acid 4977-bp deletion is associated with diminished fertility and motility of human sperm. Biology of Reproduction 52 729-736. (https://doi. org/10.1095/biolreprod52.4.729)

Kao SH, Chao HT \& Wei YH 1998 Multiple deletions of mitochondrial DNA are associated with the decline of motility and fertility of human spermatozoa. Molecular Human Reproduction 4 657-666. (https://doi. org/10.1093/molehr/4.7.657)

Karp NA, Mason J, Beaudet AL, Benjamini Y, Bower L, Braun RE, Brown SDM, Chesler EJ, Dickinson ME \& Flenniken AM et al. 2017 Prevalence of sexual dimorphism in mammalian phenotypic traits. Nature Communications 8 ncomms15475. (https://doi.org/10.1038/ ncomms15475)

Kuijper B, Lane N \& Pomiankowski A 2015 Can paternal leakage maintain sexually antagonistic polymorphism in the cytoplasm? Journal of Evolutionary Biology 28 468-480. (https://doi.org/10.1111/jeb.12582)

Kumar RS, Venkatesh M, Kumar M, Tanwar MB, Shasmsi NP, Gupta RK, Talwar SP \& Dada R 2009 Oxidative stress and sperm mitochondrial DNA mutation in idiopathic oligoasthenozoospermic men. Indian Journal of Biochemistry and Biophysics 46 172-177.

Lestienne P, Reynier P, Chretien MF, Penisson-Besnier I, Malthiery Y \& Rohmer V 1997 Oligoasthenospermia associated with multiple mitochondrial DNA rearrangements. Molecular Human Reproduction 3 811-814. (https://doi.org/10.1093/molehr/3.9.811)

Lu C, Xu M, Wang R, Qin Y, Ren J, Wu W, Song L, Wang S, Zhou Z, Shen H et al. 2015 A genome-wide association study of mitochondrial DNA in Chinese men identifies two risk single nucleotide substitutions for idiopathic oligoasthenospermia. Mitochondrion 24 87-92. (https://doi. org/10.1016/j.mito.2015.07.007)

Ma H, Gutierrez NM, Morey R, Van Dyken C, Kang E, Hayama T, Lee Y, Li Y, Tippner-Hedges R, Wolf Don P et al. 2016 Incompatibility between nuclear and mitochondrial genomes contributes to an interspecies reproductive barrier. Cell Metabolism 24 283-294. (https://doi. org/10.1016/j.cmet.2016.06.012)

Martikainen MH, Grady JP, Ng YS, Alston CL, Gorman GS, Taylor RW, McFarland R \& Turnbull DM 2017 Decreased male reproductive success in association with mitochondrial dysfunction. European Journal of Human Genetics 251162 (https://doi.org/10.1038/ejhg.2017.114)

Meiklejohn CD, Montooth KL \& Rand DM 2007 Positive and negative selection on the mitochondrial genome. Trends in Genetics 23 259-263. (https://doi.org/10.1016/j.tig.2007.03.008)

Milot E, Moreau C, Gagnon A, Cohen AA, Brais B \& Labuda D 2017 Mother's curse neutralizes natural selection against a human genetic disease over three centuries. Nature Ecology and Evolution 11400. (https://doi.org/10.1038/s41559-017-0276-6)

Montiel-Sosa F, Ruiz-Pesini E, Enríquez JA, Marcuello A, Díez-Sánchez C, Montoya J, Wallace DC \& López-Pérez MJ 2006 Differences of sperm motility in mitochondrial DNA haplogroup $U$ sublineages. Gene $\mathbf{3 6 8}$ 21-27. (https://doi.org/10.1016/j.gene.2005.09.015)

Morrow EH, Reinhardt K, Wolff JN \& Dowling DK 2015 Risks inherent to mitochondrial replacement. EMBO Reports 16 541-544. (https://doi. org/10.15252/embr.201439110)

Nakada K, Sato A, Sone H, Kasahara A, Ikeda K, Kagawa Y, Yonekawa H \& Hayashi JI 2004 Accumulation of pathogenic $\triangle \mathrm{mtDNA}$ induced deafness but not diabetic phenotypes in mito-mice. Biochemical and Biophysical Research Communications 323 175-184.

Nakada K, Sato A, Yoshida K, Morita T, Tanaka H, Inoue SI, Yonekawa H \& Hayashi JI 2006 Mitochondria-related male infertility. PNAS 103 15148-15153. (https://doi.org/10.1073/pnas.0604641103)

Patel MR, Miriyala GK, Littleton AJ, Yang H, Trinh K, Young JM, Kennedy SR, Yamashita YM, Pallanck LJ \& Malik HS 2016 A mitochondrial DNA hypomorph of cytochrome oxidase specifically impairs male fertility 
in Drosophila melanogaster. eLife 5 e16923. (https://doi.org/10.7554/ eLife.16923)

Pennell TM \& Morrow EH 2013 Two sexes, one genome: the evolutionary dynamics of intralocus sexual conflict. Ecology and Evolution $\mathbf{3}$ 1819-1834. (https://doi.org/10.1002/ece3.540)

Radzvilavicius AL, Lane N \& Pomiankowski A 2017 Sexual conflict explains the extraordinary diversity of mechanisms regulating mitochondrial inheritance. BMC Biology 1594.

Rajender S, Rahul P \& Mahdi AA 2010 Mitochondria, spermatogenesis and male infertility. Mitochondrion 10 419-428. (https://doi.org/10.1016/j. mito.2010.05.015)

Rand DM 2001 The units of selection of mitochondrial DNA. Annual Review of Ecology and Systematics 32 415-448. (https://doi.org/10.1146/ annurev.ecolsys.32.081501.114109)

Rand DM, Dorfsman M \& Kann LM 1994 Neutral and non-neutral evolution of Drosophila mitochondrial DNA. Genetics 138 741-756.

Rand DM, Haney RA \& Fry AJ 2004 Cytonuclear coevolution: the genomics of cooperation. Trends in Ecology and Evolution 19 645-653. (https:// doi.org/10.1016/j.tree.2004.10.003)

Reeve AK, Krishnan KJ \& Turnbull D 2008 Mitochondrial DNA mutations in disease, aging, and neurodegeneration. Annals of the New York Academy of Sciences 1147 21-29. (https://doi.org/10.1196/annals.1427.016)

Reinhardt K, Dowling DK \& Morrow EH 2013 Mitochondrial replacement, evolution, and the clinic. Science 341 1345-1346. (https://doi. org/10.1126/science.1237146)

Ruiz-Pesini E, Lapeña AC, Díez C, Álvarez E, Enríquez JA \& López-Pérez MJ 2000a Seminal quality correlates with mitochondrial functionality. Clinica Chimica Acta 300 97-105. (https://doi.org/10.1016/S00098981(00)00305-3)

Ruiz-Pesini E, Lapena AC, Díez-Sánchez C, Pérez-Martos A, Montoya J, Alvarez E, Díaz M, Urriés A, Montoro L, López-Pérez MJ et al. $2000 b$ Human mtDNA haplogroups associated with high or reduced spermatozoa motility. American Journal of Human Genetics 67 682-696. (https://doi.org/10.1086/303040)

Short RV 1997 The testis: the witness of the mating system, the site of mutation and the engine of desire. Acta Paediatrica 86 3-7. (https://doi. org/10.1111/j.1651-2227.1997.tb18336.x)

Shoubridge EA \& Wai T 2007 Mitochondrial DNA and the mammalian oocyte. Current Topics in Developmental Biology 77 87-111. (https:// doi.org/10.1016/S0070-2153(06)77004-1)

Smith S, Turbill C \& Suchentrunk F 2010 Introducing mother's curse: low male fertility associated with an imported mtDNA haplotype in a captive colony of brown hares. Molecular Ecology 19 36-43. (https://doi. org/10.1111/j.1365-294X.2009.04444.x)

Spiropoulos J, Turnbull DM \& Chinnery PF 2002 Can mitochondrial DNA mutations cause sperm dysfunction? Molecular Human Reproduction 8 719-721. (https://doi.org/10.1093/molehr/8.8.719)

St John JC, Jokhi RP \& Barratt CL 2001 Men with oligoasthenoteratozoospermia harbour higher numbers of multiple mitochondrial DNA deletions in their spermatozoa, but individual deletions are not indicative of overall aetiology. Molecular Human Reproduction 7 103-111. (https://doi.org/10.1093/molehr/7.1.103)

Taylor RW \& Turnbull DM 2005 Mitochondrial DNA mutations in human disease. Nature Reviews Genetics 6 389-402. (https://doi.org/10.1038/ nrg1606)

Thangaraj K, Joshi MB, Reddy AG, Rasalkar AA \& Singh L 2003 Sperm mitochondrial mutations as a cause of low sperm motility. Journal of Andrology 24 388-392. (https://doi.org/10.1002/j.1939-4640.2003. tb02687.x)

Thorburn DR 2004 Mitochondrial disorders: prevalence, myths and advances. Journal of Inherited Metabolic Disease 27 349-362. (https:// doi.org/10.1023/B:BOLI.0000031098.41409.55)

Tourmente M, Hirose M, Ibrahim S, Dowling DK, Tompkins DM, Roldan ER \& Gemmell NJ 2017 mtDNA polymorphism and metabolic inhibition affect sperm performance in conplastic mice. Reproduction 154 341-354. (https://doi.org/10.1530/REP-17-0206)
Trifunovic A, Wredenberg A, Falkenberg $M$, Spelbrink JN, Rovio AT, Bruder CE, Bohlooly-y M, Gidlöf S, Oldfors A, Wibom R et al. 2004 Premature ageing in mice expressing defective mitochondrial DNA polymerase. Nature 429 417-423. (https://doi.org/10.1038/nature02517)

Unckless RL \& Herren JK 2009 Population genetics of sexually antagonistic mitochondrial mutants under inbreeding. Journal of Theoretical Biology 260 132-136. (https://doi.org/10.1016/j.jtbi.2009.06.004)

Wade MJ 2014 Paradox of Mother's Curse and the maternally provisioned offspring microbiome. Cold Spring Harbor Perspectives in Biology 6 a017541. (https://doi.org/10.1101/cshperspect.a017541)

Wade MJ \& Brandvain Y 2009 Reversing mother's curse: selection on male mitochondrial fitness effects. Evolution 63 1084-1089. (https://doi. org/10.1111/j.1558-5646.2009.00614.x)

Wallace DC 1999 Mitochondrial diseases in man and mouse. Science 283 1482-1488. (https://doi.org/10.1126/science.283.5407.1482)

Wallace DC 2005 The mitochondrial genome in human adaptive radiation and disease: on the road to therapeutics and performance enhancement. Gene 354 169-180. (https://doi.org/10.1016/j.gene.2005.05.001)

White DJ, Wolff JN, Pierson M \& Gemmell NJ 2008 Revealing the hidden complexities of mtDNA inheritance. Molecular Ecology 17 4925-4942. (https://doi.org/10.1111/j.1365-294X.2008.03982.x)

Wolff JN \& Gemmell NJ 2013 Mitochondria, maternal inheritance, and asymmetric fitness: why males die younger. BioEssays 35 93-99. (https:// doi.org/10.1002/bies.201200141)

Wolff JN, Ladoukakis ED, Enríquez JA \& Dowling DK 2014 Mitonuclear interactions: evolutionary consequences over multiple biological scales. Philosophical Transactions of the Royal Society B 369 20130443. (https:// doi.org/10.1098/rstb.2013.0443)

Wolff JN, Pichaud N, Camus MF, Côté G, Blier PU \& Dowling DK 2016a Evolutionary implications of mitochondrial genetic variation: mitochondrial genetic effects on OXPHOS respiration and mitochondrial quantity change with age and sex in fruit flies. Journal of Evolutionary Biology 29 736-747. (https://doi.org/10.1111/jeb.12822)

Wolff JN, Tompkins DM, Gemmell NJ \& Dowling DK 2016b Mitonuclear interactions, mtDNA-mediated thermal plasticity, and implications for the Trojan Female Technique for pest control. Scientific Reports 630016. (https://doi.org/10.1038/srep30016)

Wolff JN, Gemmell NJ, Tompkins DM \& Dowling DK 2017 Introduction of a male-harming mitochondrial haplotype via 'Trojan Females' achieves population suppression in fruit flies. elife 6 e23551.

Xu H, DeLuca SZ \& O'Farrell PH 2008 Manipulating the metazoan mitochondrial genome with targeted restriction enzymes. Science $\mathbf{3 2 1}$ 575-577. (https://doi.org/10.1126/science.1160226)

Yee WK, Sutton KL \& Dowling DK 2013 In vivo male fertility is affected by naturally occurring mitochondrial haplotypes. Current Biology 23 R55-R56. (https://doi.org/10.1016/j.cub.2012.12.002)

Yu X, Gimsa U, Wester-Rosenlöf L, Kanitz E, Otten W, Kunz M \& Ibrahim SM 2009 Dissecting the effects of mtDNA variations on complex traits using mouse conplastic strains. Genome Research 19 159-165. (https://doi.org/10.1101/gr.078865.108)

Zhang H, Guillaume F \& Engelstädter J 2012 The dynamics of mitochondrial mutations causing male infertility in spatially structured populations. Evolution 66 3179-3188. (https://doi.org/10.1111/j.15585646.2012.01675.x)

Zucker I \& Beery AK 2010 Males still dominate animal studies. Nature 465 690-690. (https://doi.org/10.1038/465690a)

Received 3 October 2017

First decision 17 November 2017

Revised manuscript received 13 October 2017

Accepted 18 January 2018 